\title{
Integrating Answer Set Programming and Constraint Logic Programming
}

\author{
Veena S. Mellarkod and Michael Gelfond and Yuanlin Zhang \\ \{veena.s.mellarkod,mgelfond,yzhang\}@cs.ttu.edu \\ Texas Tech University
}

Dedicated to Victor Marek on his 65th birthday

\begin{abstract}
We introduce a knowledge representation language $\mathcal{A C}(\mathcal{C})$ extending the syntax and semantics of ASP and CR-Prolog, give some examples of its use, and present an algorithm, $\mathcal{A C}$ solver, for computing answer sets of $\mathcal{A C}(\mathcal{C})$ programs. The algorithm does not require full grounding of a program and combines "classical" ASP solving methods with constraint logic programming techniques and CR-Prolog based abduction. The $\mathcal{A C}(\mathcal{C})$ based approach often allows to solve problems which are impossible to solve by more traditional ASP solving techniques. We belief that further investigation of the language and development of more efficient and reliable solvers for its programs can help to substantially expand the domain of applicability of the answer set programming paradigm.
\end{abstract}

\section{Introduction}

The work presented in this paper is aimed at further development of declarative programming paradigm based on Answer Set Prolog (ASP) (Gelfond \& Lifschitz 1991; Baral 2003) and its extensions. The language has roots in research on non-monotonic logic and semantics of default negation of Prolog (for more details, see (Marek \& Truszczynski 1993)). An ASP program $\Pi$ is a collection of rules of the form

$$
l_{1} \text { or } \ldots \text { or } l_{k} \leftarrow l_{k+1}, \ldots, l_{n}, \text { not } l_{n+1}, \ldots, \text { not } l_{m}
$$

where $l$ 's are literals (statements of the form $p(\bar{t})$ and $\neg p(\bar{t})$ ) over some signature $\Sigma$. Expression on the left hand side of $\leftarrow$ is called the head of the rule; that on the right hand side is called the rule's body. Note that both the body and the head of the rule can be empty. If the body of a rule is empty then the $\leftarrow$ is omitted and the rule is referred to as a fact. Connectives or and not are referred to as epistemic disjunction and default negation respectively; $\neg$ is often referred to as classical or strong negation. An ASP program $\Pi$ can be viewed

Copyright (C) 2007, V. Mellarkod, M. Gelfond and Y. Zhang. All rights reserved. as a specification for the sets of beliefs to be held by a rational reasoner associated with $\Pi$. Such sets, called answer sets of $\Pi$, are represented by collections of ground literals. A rule (1) is viewed as a constraint which says that if literals $l_{k+1}, \ldots, l_{n}$ belong to an answer set $A$ of $\Pi$ and none of the literals $l_{n+1}, \ldots, l_{m}$ belong to $A$ then $A$ must contain at least one of the literals $l_{1}, \ldots, l_{k}$. To form answer sets of $\Pi$, the reasoner must satisfy $\Pi$ 's rules together with the rationality principle which says: "Believe nothing you are not forced to believe".

Given a computational problem $P$, an ASP programmer

- Expresses information relevant to the problem in the language of ASP;

- Reduces $P$ to a query $Q$ requesting computation of (parts of) answer sets of $\Pi$.

- Uses inference engine, i.e., a collection of reasoning algorithms, to solve $Q$.

There is a number of inference engines available to an ASP programmer. If the corresponding program does not contain disjunction, classical negation or rules with empty heads and is acyclic (Apt \& Bezem 1991), i.e., only allows naturally terminating recursion, then the classical SLDNF-resolution of Prolog (Clark 1978) and its variants (Chen, Swift, \& Warren 1995) or fix-point computations of deductive databases (possibly augmented by constraint solving algorithms as in (Van Hentenryck 1989; Jaffar et al. 1992; Marriott \& Stuckey 1998)) can be used to answer the query $Q$. Presently, there are multiple applications of solving various computational problems using these methods. In the last decade we have witnessed the coming of age of inference engines aimed at computing the answer sets of Answer Set Prolog programs (Niemela \& Simons 1997; Niemela, Simons, \& Soininen 2002; Leone et al. 2006; Eiter et al. 1997; Gebser et al. 2007; Giunchiglia, Lierler, \& Maratea 2006). These engines are often referred to as answer set solvers. Normally they start their work with grounding the program, i.e., instantiating its variables by ground terms. The resulting program has the same answer sets as the original one but is essentially propositional. The grounding techniques employed by answer set solvers are rather 
sophisticated. Among other things they utilize algorithms from deductive databases, and require a good understanding of the relationship between various semantics of logic programming. The answer sets of the grounded program are often computed using substantially modified and expanded satisfiability checking algorithms. Another approach reduces the computation of answer sets to (possibly multiple) calls to existing satisfiability solvers (Babovich \& Maratea 2004; Giunchiglia, Lierler, \& Maratea 2006; Lin \& Zhao 2004).

The programming methodology based on the use of ASP solvers was originally advocated in (Marek \& Truszczynski 1999; Niemela 1999). It proved to be useful for finding solutions to a variety of programming tasks, ranging from building decision support systems for the Space Shuttle (Balduccini, Gelfond, \& Nogueira 2006) and program configuration (Soininen \& Niemella 1998), to solving problems arising in bio-informatics (Baral et al. 2004), zoology and linguistics (Brooks et al. 2005). Though positive, this experience allowed to identify a number of problems and inadequacies of the ASP approach to declarative programming.

First it became clear that for a number of tasks which require the use of ASP solvers these solvers are not sufficiently efficient. This becomes immediately obvious if the program contains variables ranging over large domains. Even though ASP solvers use intelligent grounding optimization techniques, ground instantiations of such a program can still be huge, which can cause both memory and time problems and make ASP solvers practically useless. The problem was partially addressed in (Baselice, Bonatti, \& Gelfond 2005; Mellarkod \& Gelfond 2007) where the language of ASP and its reasoning mechanism were extended to partially avoid grounding of variables ranging over the large domains and to replace such grounding with the use of constraint solving techniques. In (Mellarkod \& Gelfond 2007) an algorithm was implemented for a language allowing so called difference constraints which substantially expanded the scope of applicability of the ASP paradigm. In this paper we further expand this work by designing a more powerful extension $\mathcal{A C}(\mathcal{C})$ of ASP and define an algorithm, $\mathcal{A C}$ solver, for computing answer sets of programs in the new language. The algorithm combines "classical" ASP solving methods with constraint satisfaction techniques and SLDNF resolution. The prototype implementations of the solver were tested on a number of examples including planning and scheduling tasks related to the space shuttle. The results are mostly positive and, since the prototype implementation allows many natural improvements, we have no doubt that a fully adequate solution will be produced soon. (It is worth noting that standard ASP methods are fully inadequate for this task).

The second difficulty of using ASP for a number of ap- plications was related to insufficient expressive power of the language. For instance, in a typical diagnostic task one often needs to explain unusual behavior of a system manifested by the inconsistency of an ASP program encoding its normal behavior. This requires the ability to naturally mix the computation of answer sets of a program with some form of abductive reasoning. We were not able to find a way to utilize the existing abductive logic programming systems for such tasks, and opted for an introduction of a new language, CR-Prolog (Balduccini \& Gelfond 2003; Balduccini 2007), which is capable of expressing rare events which are ignored during a normal computation and only used if needed to restore consistency of the program. Consider, for instance, a program $\Pi_{0}$ consisting of regular ASP rules

$\neg p \leftarrow \operatorname{not} p$
$q \leftarrow \neg p$

which say that $p$ is normally believed to be false, and that if $p$ is believed to be false then $q$ must be believed to be true. The program has a unique answer set $\{\neg p, q\}$. Now let us expand $\Pi_{0}$ by a consistency restoring rule (CR-rule)

$p \leftarrow+$

which says that $p$ is possible but so rare that it can be ignored during the reasoning process unless it is needed for restoring consistency. The resulting program $\Pi_{1}$ still has one answer set $\{\neg p, q\}$. The CR-rule above remains unused. The situation changes if we expand $\Pi_{1}$ by a new fact

$\neg q$

Since regular rules of the new program $\Pi_{2}$ are inconsistent, the reasoner associated with the program is forced to use the CR-rule. The resulting answer set is $\{p, \neg q\}$.

The expressive power and reasoning ability of CRProlog proved to be useful in many situations beyond diagnostic reasoning. CR-Prolog was also successfully used in planning to produce higher quality plans than regular ASP (Balduccini 2004), for reasoning about intentions, reasoning with weak constraints a la DLV, etc. So we expand $\mathcal{A C}(\mathcal{C})$ by $\mathrm{CR}$-rules and show an example of their use adding CR-Prolog abduction to the plethora of reasoning techniques discussed above.

\section{Syntax and Semantics of $\mathcal{A C}(\mathcal{C})$ 2.1 Answer Set Prolog}

Recall that terms, literals, and rules of program $\Pi$ with signature $\Sigma(\Pi)$ are called ground if they contain no variables and no symbols for arithmetic functions. A program is called ground if all its rules are ground. In 
this section we briefly review the semantics of ground programs of Answer Set Prolog.

Consistent sets of ground literals over $\Sigma$, containing all arithmetic literals which are true under the standard interpretation of their symbols, are called partial interpretations of $\Sigma$. Expressions $l$ and not $l$ where $l$ is a literal are called extended literals. We say that $l$ is true in a partial interpretation $S$ if $l \in S$; not $l$ is true in $S$ if $l \notin S$; disjunction $l_{1}$ or ... or $l_{k}$ is true in $S$ if at least one of its members is true in $S$. S satisfies a logic programming rule (1) if $S$ satisfies its head or does not satisfy its body.

The answer set semantics of a logic program $\Pi$ assigns to $\Pi$ a collection of answer sets - partial interpretations of the signature $\Sigma(\Pi)$ corresponding to the possible sets of beliefs which can be built by a rational reasoner on the basis of the rules of $\Pi$ and the rationality principle. The precise definition of answer sets will be first given for programs whose rules do not contain default negation. Let $\Pi$ be such a program and let $S$ be a partial interpretation of $\Sigma(\Pi)$.

Definition 1 (Answer set-part one)

A partial interpretation $S$ of $\Sigma(\Pi)$ is an answer set of $\Pi$ if $S$ is minimal (in the sense of set-theoretic inclusion) among the partial interpretations satisfying the rules of $\Pi$.

(Note that the rationality principle is captured in this definition by the minimality requirement).

To extend the definition of answer sets to arbitrary programs, take any program $\Pi$, and let $S$ be a partial interpretation of $\Sigma(\Pi)$. The reduct, $\Pi^{S}$, of $\Pi$ relative to $S$ is the set of rules

$$
l_{1} \text { or } \ldots \text { or } l_{k} \leftarrow l_{k+1}, \ldots, l_{m}
$$

for all rules (1) in $\Pi$ such that $\left\{l_{m+1}, \ldots, l_{n}\right\} \cap S=\emptyset$. Thus $\Pi^{S}$ is a program without default negation.

Definition 2 (Answer set - part two)

A partial interpretation $S$ of $\Sigma(\Pi)$ is an answer set of $\Pi$ if $S$ is an answer set of $\Pi^{S}$.

(Here the rationality principle is captured by the fixpoint condition above). A program is called consistent if it has an answer set.

\subsection{The language $\mathcal{A C}(\mathcal{C})$}

Now we will describe the syntax and informal semantics of the language $\mathcal{A C}(\mathcal{C})$. First let us recall some necessary terminology.

By sort we mean a non-empty countable collection of strings over some fixed alphabet. Strings of sort $S_{i}$ will be referred to as object constants of $S_{i}$. A sorted signature, $\Sigma$, is a collection of sorts, properly typed predicate and function symbols, and variables. Each variable, $X$, takes on values from a unique sort denoted by $\operatorname{sort}(X)$. When needed we assume that $\Sigma$ contains standard numerical sorts of natural numbers, integers, rational numbers, etc. as well as standard numerical functions and relations such as,,$+->,<$ etc.. Terms, literals, and extended literals of $\Sigma$ are defined as usual. Rules of the language are expressions of the form (1) where l's are literals which may possibly contain variables. In the standard ASP semantics a rule with variables is viewed as a shorthand for a collection of its ground instantiations. The $\mathcal{A C}(\mathcal{C})$ interpretation of rules with variables is different and allows the construction of solvers which will not require a complete grounding of the program. To achieve this goal we first expand the language of ASP by:

- Dividing sorts of $\Sigma$ into regular and constraint. Constraint sorts will be declared by an expression \#csort. For instance a sort time of integers, say, between 0 and 1000 can be declared to be a constraint sort by statement

\# csort(time).

Undeclared sorts are assumed to be regular. Intuitively a sort is declared to be a constraint sort if it is a large (often numerical) set with primitive constraint relations like $\leq$ defined between its elements. Grounding constraint variables, i.e., variables ranging over constraint sorts, would normally lead to huge grounded program. This is exactly what should be avoided by the $\mathcal{A C}(\mathcal{C})$ solvers. The $\mathcal{A C}(\mathcal{C})$ solvers will only ground variables ranging over regular sorts (regular variables).

- Dividing predicates of the language into four types: regular, constraint, defined and mixed. Intuitively, regular predicates denote relations among objects of regular sorts; constraint predicates denote primitive numerical relations among objects of constraint sorts; defined predicates are defined in terms of constraint predicates; mixed predicates denote relations between objects which belong to regular sorts and those which belong to constraint ones. They are not defined by the rules of the program and are similar to abducible relations of abductive logic programming.

Consider for instance a regular sort step $=\{0 . .30\}$ used to denote steps of a trajectory of some acting agent, and constraint sort time $=\{0 . .1000\}$ used to denote actual time (say in minutes). The relation at $(S, T)$ which holds iff step $S$ of the trajectory was executed at time $T$ is a typical example of mixed relation. The corresponding declaration of this relation will be given by statement

\#mixed at(step, time).

Without loss of generality, we will assume that in any mixed predicate $m$ of $\Pi$ 's signature, constraint parameters follow regular parameters, i.e., every mixed 
atom formed by $m$ can be written as $m\left(\bar{t}_{r}, \bar{t}_{c}\right)$ where $\bar{t}_{r}$ and $\bar{t}_{c}$ are the lists of regular and constraint terms respectively. According to our semantics a mixed predicate can be viewed as a function whose domain and range are collections of properly typed vectors of regular and constraint terms respectively. Hence $m\left(\bar{t}_{r}, \bar{t}_{c}\right)$ can be written as $m\left(\bar{t}_{r}\right)=\bar{t}_{c}$. If the range of $m$ is boolean we write $m\left(\bar{t}_{r}\right)$ instead of $m\left(\bar{t}_{r}\right)=$ true and $\neg m\left(\bar{t}_{r}\right)$ instead of $m\left(\bar{t}_{r}\right)=$ false.

Now let acceptable_time $(T)$ be true iff time $T$ belongs to the interval $[10,20]$ or $[100,120]$. It is natural to view this predicate as defined. The corresponding declaration is as follows:

\#defined acceptable_time(time).

$\mathcal{A C}(\mathcal{C})$ does not require special declaration for the constraint predicates. They are to be specified in the parameter $C$ of the language. Non-constraint predicate without a declaration is considered to be regular. A literal formed by a regular predicate will be called regular literal. Similarly for constraint, defined, and mixed literals.

Definition 3 (Syntax of $\mathcal{A C}(\mathcal{C})$ )

$A n \mathcal{A C}(\mathcal{C})$ rule over signature $\Sigma$ is a statement of the form:

$$
h_{1} \text { or } \ldots \text { or } h_{k} \leftarrow l_{1}, \ldots, l_{m}, \text { not } l_{m+1}, \ldots, \text { not } l_{n}
$$

such that

- if $k>1$ then $h_{1}, \ldots, h_{k}$ are regular literals,

- if $k=1$ then $h_{1}$ is a regular or defined literal.

- $l_{1}, \ldots, l_{n}$ are arbitrary literals of $\Sigma$.

An $\mathcal{A C}(\mathcal{C})$ program $\Pi$ consists of a signature $\Sigma(\Pi)$ and a collection of $\mathcal{A C}(\mathcal{C})$ rules over this signature.

Classification of literals of the signature $\Sigma(\Pi)$ of an $\mathcal{A C}(\mathcal{C})$ program $\Pi$ allows partitioning $\Pi$ into three parts:

- Regular parts, $\Pi_{R}$, consisting of rules built from regular literals.

- Defined part, $\Pi_{D}$, consisting of rules whose heads are defined literals.

- Middle part, $\Pi_{M}$, consisting of all other rules of $\Pi$.

Elements of $\Pi_{R}, \Pi_{D}$ and $\Pi_{M}$ are called regular rules, defined rules, and middle rules respectively. Note that a standard (ground) $A S P$ program $\Pi$ is also an $\mathcal{A C}(\mathcal{C})$ program in which all the predicates are defined as regular.

\subsection{Semantics of $\mathcal{A C}(\mathcal{C})$}

First we will need some terminology. Let $r$ be a rule of an $\mathcal{A C}(\mathcal{C})$ program $\Pi$. A ground instance of $r$ is obtained from $r$ by:

1. replacing variables of $r$ by ground terms from the respective sorts;
2. replacing all numerical terms by their values.

An $A S P$ program ground(П) consisting of all ground instances of all rules in $\Pi$ is called the ground instantiation of $\Pi$.

A consistent set $S$ of ground literals over the signature $\Sigma(\Pi)$ is called a partial interpretation of an $\mathcal{A C}(\mathcal{C})$ program $\Pi$ if it satisfies the following conditions:

1. A constraint literal $l \in S$ iff $l$ is true under the intended interpretation of its symbols;

2. For every mixed predicate $m\left(\bar{X}_{r}, \bar{Y}_{c}\right)$ and every ground instantiation $\bar{t}_{r}$ of $\bar{X}_{r}$, there is a unique ground instantiation $\bar{t}_{c}$ of $\bar{X}_{c}$ such that $m\left(\bar{t}_{r}, \bar{t}_{c}\right) \in S$.

Definition 4 (Answer Sets of $\mathcal{A C}(\mathcal{C})$ )

$A$ partial interpretation $S$ of an $\mathcal{A C}(\mathcal{C})$ program $\Pi$ is called an answer set of $\Pi$ if there is a set $M$ of ground mixed literals such that $S$ is an answer set of the $A S P$ program ground $(\Pi) \cup M^{1}$.

Let us illustrate the definition by the following example.

Example $1[\mathcal{A C}(\mathcal{C})$ program and its answer sets] Let $P$ be an $\mathcal{A C}(\mathcal{C})$ program with sorts

time (0..1000).

step $(0 . .1)$.

$\operatorname{action}(a)$.

fluent $(f)$.

constraint relation $\leq$ defined on time, declarations

\# csort(time).

\#defined acceptable_time(time).

\#mixed at (step, time).

\#regular occurs(action, step).

\# regular holds(fluent, step).

\# regular next(step, step).

and rules

acceptable_time $(T) \leftarrow 10 \leq T \leq 20$.

acceptable_time $(T) \leftarrow 100 \leq T \leq 120$.

$\neg \operatorname{occurs}(A, S) \leftarrow \operatorname{at}(S, T)$, not acceptable_time $(T)$.

$\operatorname{next}(1,0)$.

$\begin{aligned} \operatorname{holds}\left(f, S^{\prime}\right) \leftarrow \quad & \operatorname{occurs}(a, S), \\ & \operatorname{next}\left(S^{\prime}, S\right) .\end{aligned}$

$\operatorname{occurs}(a, 0)$.

\footnotetext{
${ }^{1}$ Note that since ground $(\Pi) \cup M$ contains no type declarations all its predicates are considered to be regular and hence it can be viewed as an ASP program whose answer sets are given by definitions 1 and 2 .
} 
The first two rules comprise the defined part, $P_{D}$, of the program. Its middle part, $P_{M}$, consists of the third rule. The remaining rules form $P$ 's regular part, $P_{R}$.

Let $I=[10,20] \cup[100,120]$ and $t_{0}, t_{1}$, and $t_{2}$ be arbitrary elements of time such that $t_{0}, t_{1} \in I$ and $t_{2} \notin I$. Let $A_{1}$ be a collection of atoms consisting of specification of sorts, step $(0)$, step $(1), \operatorname{action}(a)$, etc, and atoms

$\operatorname{at}\left(0, t_{0}\right), a t\left(1, t_{1}\right)$,

next $(1,0)$, occurs $(a, 0)$,

holds $(f, 1)$,

acceptable_time $(t)$ for every $t \in I$.

Let $A_{2}=\left(A_{1} \backslash\left\{a t\left(1, t_{1}\right)\right\}\right) \cup\left\{a t\left(1, t_{2}\right), \neg\right.$ occurs $\left.(a, 1)\right\}$. It is not difficult to check that $A_{1}$ and $A_{2}$ are answer sets of $P$.

The syntax and semantics of $\mathcal{A C}(\mathcal{C})$ can be easily extended to allow the use of consistency restoring rules. To do that we simply need to replace the words " $A S P$ program" in Definition 4 by "CR-Prolog program".

\section{Computing answer sets of $\mathcal{A C}(\mathcal{C})$ programs}

In this section we present a (somewhat simplified) version of $\mathcal{A C}$ solver - an algorithm for computing answer sets of $\mathcal{A C}(\mathcal{C})$ programs. $\mathcal{A C}$ solver takes as an input a program $\Pi$ which satisfies the following conditions:

1. $\Pi$ contains no regular variables. (We refer to such programs as r-ground.)

2. П contains neither or nor $\neg$.

3. Every mixed atom of $\Pi$ has a form $m\left(\bar{t}_{r}, \bar{X}\right)$ where $\bar{X}$ is a list of constraint variables.

4. If an atom occurs in the head of a middle rule it does not occur in the head of any other rule.

5. A middle rule of $\Pi$ contains at most one occurrence of a defined atom and no occurrences of constraint atoms.

The restrictions are not too severe. Regular variables may be removed by a grounding process. (In our implementations this is done by a modification of grounding algorithm lparse (Niemela, Simons, \& Soininen 2002)). Classical negation can be eliminated from the program by viewing $\neg p$ as a new predicate symbol and adding constraints $\leftarrow p(\bar{t}), \neg p(\bar{t})$. Mixed atom $m\left(\bar{t}_{r}, t_{c}^{1}, \ldots, t_{c}^{n}\right)$ can be replaced by $m\left(\bar{t}_{r}, X_{1}, \ldots, X_{n}\right)$ and $X_{1}=t_{c}^{1}, \ldots, X_{n}=t_{c}^{n}$. Middle rules $p(\bar{t}) \leftarrow B_{1}$ and $p(\bar{t}) \leftarrow B_{2}$ can be replaced by $p_{1}(\bar{t}) \leftarrow B_{1}, p_{2}(\bar{t}) \leftarrow B_{2}$, $p(\bar{t}) \leftarrow p_{1}(\bar{t})$ and $p(\bar{t}) \leftarrow p_{2}(\bar{t})$, where $p_{1}$ and $p_{2}$ are new regular predicate symbols. (Since only regular predicates can occur in the heads of middle rules the last two rules are regular.) A middle rule $p(\bar{t}) \leftarrow B_{1}, B_{2}$, where $B_{2}$ is the collection of the defined and constraint extended literals of the rule, can be replaced by $d(\bar{X}) \leftarrow B_{2}$ and $p(\bar{t}) \leftarrow B_{1}, d(\bar{X})$. (Here $\bar{X}$ is the list of variables of $B_{2}$.) Of course, the absence of disjunction is a serious limitation, but this restriction is made only to simplify the presentation.

Now we give a (necessarily sketchy) description of several functions used by $\mathcal{A C}$ solver. Throughout this section we conform to the common terminology and refer to extended literals of the language as literals. Literal not not $l$ will be identified with $l$. Literals $p(\bar{t})$ and not $p(\bar{t})$ will be called contrary. We say that a set $B$ of literals falsifies rule $r$ of $\Pi$ if the body of $r$ contains a literal contrary to some literal of $B$. The functions and the algorithm will be illustrated using program $\operatorname{gr}(P)-$ the result of grounding the regular variables of program $P$ from Example 1.

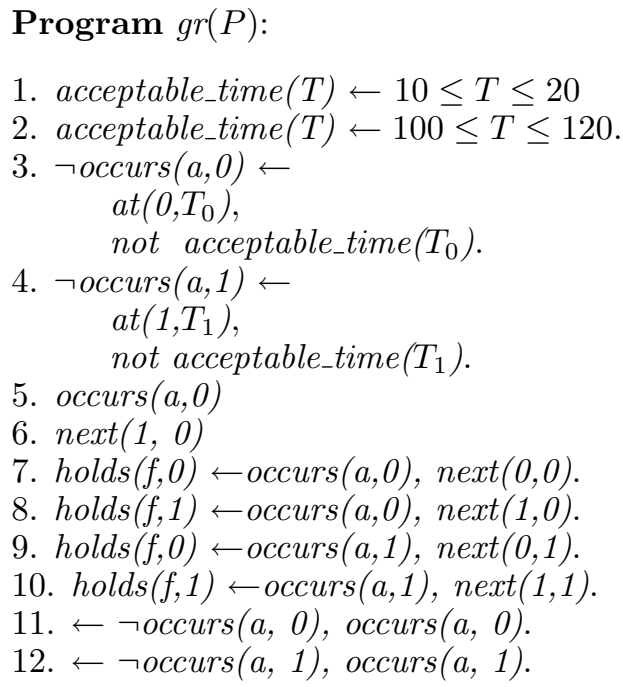

5. $\operatorname{occurs}(a, 0)$

6. $\operatorname{next}(1,0)$

7. holds $(f, 0) \leftarrow \operatorname{occurs}(a, 0), \operatorname{next}(0,0)$.

8. holds $(f, 1) \leftarrow \operatorname{occurs}(a, 0), \operatorname{next}(1,0)$.

9. holds $(f, 0) \leftarrow \operatorname{occurs}(a, 1), \operatorname{next}(0,1)$.

10. holds $(f, 1) \leftarrow \operatorname{occurs}(a, 1), \operatorname{next}(1,1)$.

11. $\leftarrow \neg \operatorname{occurs}(a, 0), \operatorname{occurs}(a, 0)$.

$12 . \leftarrow \neg \operatorname{occurs}(a, 1), \operatorname{occurs}(a, 1)$.

(Note that last two rules are the result of elimination of classical negation).

\section{Function $C n$}

Function $C n\left(\Pi_{R} \cup \Pi_{M}, B\right)$ computes the consequences of a set of ground regular literals $B$ under $\Pi_{R} \cup \Pi_{M}$. It is similar to the corresponding functions of the regular ASP solvers (e.g., expand of Smodels). $C n$ is defined in terms of two auxiliary functions: lower bound, $l b\left(\Pi_{R} \cup\right.$ $\left.\Pi_{M}, B\right)$, and upper bound, $u b\left(\Pi_{R} \cup \Pi_{M}, B\right)$. The former computes the minimal set $X$ of literals containing $B$ and closed w.r.t. the following rules

1. If $r \in \Pi_{R}$ and the body of $r$ is satisfied by $B$, then so is the head of $r$.

2 . If $r$ is the only rule of $\Pi_{R} \cup \Pi_{M}$ whose body is not falsified by $X$ and if head $(r) \in X$, then regular literals of the body of $r$ are in $X$.

3. If $\left(\right.$ not $\left.l_{0}\right) \in X,\left(l_{0} \leftarrow B_{1}, l, B_{2}\right) \in \Pi_{R}$, and $B_{1}, B_{2} \subseteq$ $X$, then not $l \in X$.

4. If there is no rule with head $l_{0}$, or the body of every rule of $\Pi_{R} \cup \Pi_{M}$ with head $l_{0}$ is falsified by $\mathrm{X}$, then not $l_{0} \in X$.

Function $u b\left(\Pi_{R} \cup \Pi_{M}, B\right)$ returns the answer set $X$ of a definite ground program $\alpha\left(\Pi_{R} \cup \Pi_{M}, B\right)$ obtained by: 
1. Removing all rules of $\Pi_{R} \cup \Pi_{M}$ whose bodies are falsified by $B$.

2. Removing all rules of $\Pi_{R} \cup \Pi_{M}$ such that not head $(r) \in B$.

3. Removing all literals of the form not $p(\bar{t})$ from the rules of $\Pi_{R} \cup \Pi_{M}$.

4. Removing all constraint, defined, and mixed literals from the rules of $\Pi_{M}$.

Now let

$X=l b\left(\Pi_{R} \cup \Pi_{M}, B\right)$ and

$Y=\left\{\operatorname{not} p(\bar{t}): p(\bar{t}) \in u b\left(\Pi_{R} \cup \Pi_{M}, X\right)\right\}$. Then

$$
C n\left(\Pi_{R} \cup \Pi_{M}, B\right)=X \cup Y .
$$

One can check that $C n\left(\operatorname{gr}\left(P_{R} \cup P_{M}\right), \emptyset\right)$ returns the set $S_{0}=\{$ next $(1,0)$, holds $(f, 1), \operatorname{occurs}(a, 0)$, not next $(0,0)$, not next $(0,1), \quad$ not next $(1,1), \quad$ not holds $(f, 0)$, not $\operatorname{occurs}(a, 1)$, not $\neg \operatorname{occurs}(a, 0)\}$.

\section{Function query}

For simplicity of exposition, we limit ourselves to programs whose mixed predicates have one regular and one constraint parameter. We also assume that our programs contain no occurrences of negated mixed literals.

For every pair $\langle m, t\rangle$ where $m$ is a mixed predicate and $t$ is a possible value of its regular parameter, we assign a unique constraint variable $X$ called the value variable of $\langle m, t\rangle$. An $\mathcal{A C}$ solver looks for the assignment of a value, $v$ to the value variable $V$ of $\langle m, t\rangle$ such that $m(t, v)$ could be included in the corresponding answer set of $\Pi$. Intuitively, function query $(\Pi, B)$ returns the set of constraints on these variables that have to be satisfied in order for $B$ to satisfy the rules of $\Pi$. To be precise we'll need the following definitions.

An $r_{-}$ground middle rule $r$ is called active w.r.t. a set of ground regular literals $B$ if $B$ contains all the regular literals of the body of $r$. Program $p e(\Pi, B)$ is obtained from $\Pi_{M}$ by

1. Removing all rules which are not active w.r.t. $B$.

2. Removing every rule $r$ such that head $(r) \notin B$ and $n o t$ head $(r) \notin B$.

3. Removing regular literals from all the remaining rules.

Function query $(\Pi, B)$ starts with computing $p e(\Pi, B)$. Suppose that $B=\{p 1$, not $p 2, p 3\}$ and that $p e(\Pi, B)$ for some program $\Pi$ returns the program

$p 1 \leftarrow m 1(t 1, X 1), m(t 2, X 2), d 1(X 1, X 2)$.

$p 2 \leftarrow m 3(t 3, X 3)$, not $d 2(X 3)$.

$p 3 \leftarrow m 4(t 4, X 4)$, not $d 3(X 4)$.

$\leftarrow m 1(t 1, X 5), d 4(X 5)$.

where $p$ 's are regular, $m$ 's are mixed and $d$ 's are defined predicates. (We assume that variables of the program are renamed apart, i.e., no variable occurs in two rules of the program.) Function query will, whenever possible, unify mixed atoms of $p e(\Pi, B)$, and apply the resulting substitution to the program's rules. This will replace the last rule of the program by

$\leftarrow m 1(t 1, X 1), d 4(X 1)$

(Strictly speaking, variables $X 1-X 5$ would be replaced by the corresponding value variables but we will not do it here). Recall that according to our assumption about possible input of $\mathcal{A C}$ solver there are no other rules with $p 1$ in the head. Similarly for $p 2$ and $p 3$. Since we are looking for an answer set of $\Pi$ containing $B$ and $p 1 \in B$, we need to justify $p 1$. This can be done only by finding a solution of constraint $d 1(X 1, X 2)$. To justify (not $p 2) \in$ $B$ we need to find $X 3$ such that $d 2(X 3)$. Justification of $p 3 \in B$ is given by a solution of constraint not $d 3(X 4)$. Finally, to satisfy the last rule, $X 1$ must be a solution of not $d 4(X 1)$. Not surprisingly, function query returns a constraint

$$
d 1(X 1, X 2) \wedge d 2(X 3) \wedge \operatorname{not} d 3(X 4) \wedge \text { not } d 4(X 1)
$$

where variables range over their respective sorts. (Note that allowing multiple defined and constraint literals in the body of the rules will only slightly complicate the formation of the desired query.)

Consider for instance program $\operatorname{gr}(P)$ defined above and the set $S_{0}$ returned by $C n\left(\operatorname{gr}\left(P_{M} \cup P_{M}\right), \emptyset\right)$, and compute query $\left(\operatorname{gr}(P), S_{0}\right)$. The program has two middle rules $(3)$ and (4) which are both active w.r.t. $S_{0}$. Since neither $\neg \operatorname{occurs}(a, 1)$ nor not $\neg \operatorname{occur} s(a, 1)$ is in $S_{0}$, function $\operatorname{pe}\left(\operatorname{gr}(\Pi)_{M}, S_{0}\right)$ will return

$$
\begin{aligned}
\neg \operatorname{occurs}(a, 0) \leftarrow \quad & \text { at }\left(0, T_{0}\right), \\
& \text { not acceptable_time }\left(T_{0}\right) .
\end{aligned}
$$

Since $($ not $\neg$ occurs $(a, 0)) \in S_{0}$, function query will return

$$
\text { acceptable_time }\left(T_{0}\right)
$$

\section{Function $c_{-s o l v e}$}

By a constraint program we mean a collection of defined rules formed by defined literals and primitive constraints. A query is a conjunction of defined literals. Function $c_{-}$solve $(\Pi, Q)$ takes as an input a constraint program $\Pi$ and a query $Q$. The function returns a pair ( $C$, true) where $C$ is a consistent set of primitive constraints, such that for any solution $\gamma$ of $C, \gamma(Q)$ is a consequence of $\Pi$. If no such $C$ exists the function returns false.

In what follows, we will assume the existence of such a function. There are, of course, many practical systems which implement such functions for various classes of queries and constraint programs (Van Hentenryck 1989; Jaffar et al. 1992; Jaffar \& Maher 1994; SICStus Prolog 2007). 


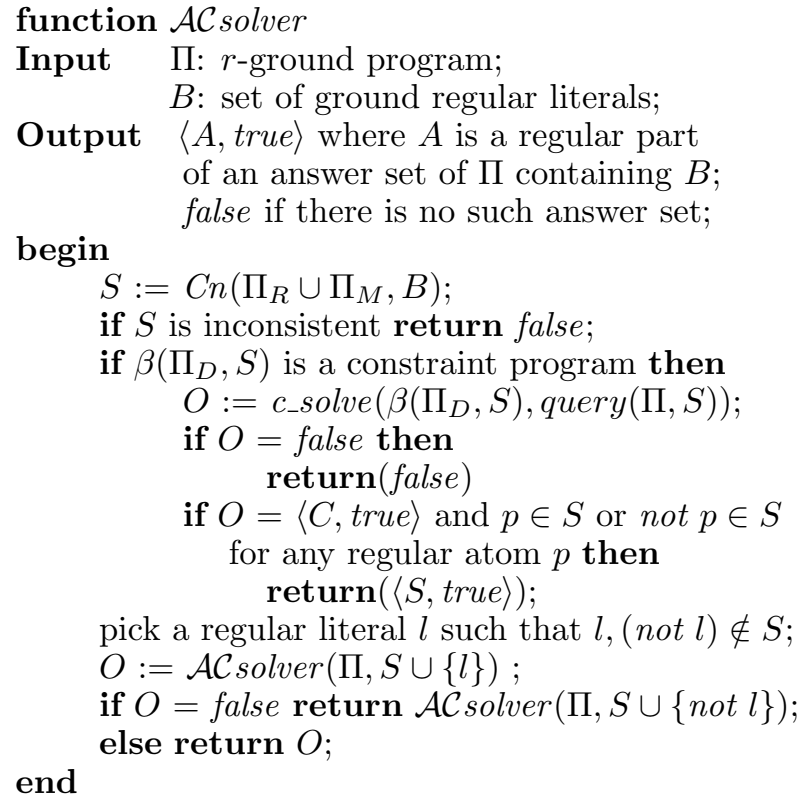

Figure 1: The $\mathcal{A C}$ solver algorithm

Finally, by $\beta(\Pi, B)$ where $B$ is a set of regular ground literals, we will denote the program obtained from $\Pi$ by (a) removing the rules whose body is falsified by $B$, and (b) removing literals of $B$ from the remaining rules of the program.

\section{Function $\mathcal{A C}$ solver}

Now we are ready to present the main function (see Figure 1), $\mathcal{A C}$ solver, which takes an $r$-ground $\mathcal{A C}(\mathcal{C})$ program $\Pi$ and a set $B$ of ground regular literals as inputs and returns an answer set of $\Pi$ containing $B$. If no such answer set exists the program returns false. Of course the actual output will be smaller. Normally we are only interested in the regular parts of $\Pi$ 's answer sets. The algorithm can be easily expended to return a solution of the set $C$ of constraints returned by $c_{-}$solve. If required, this information can be used to output relevant mixed and defined literals.

Let us trace this algorithm for $\mathcal{A C}$ solver $(\operatorname{gr}(P), \emptyset)$. We already computed the value, $S_{0}$ of $\operatorname{Cn}\left(\operatorname{gr}\left(P_{R} \cup P_{M}\right), \emptyset\right)$. $S_{0}$ is consistent, $\beta\left(\operatorname{gr}(P)_{D}, S_{0}\right)=\operatorname{gr}(P)_{D}$ and, as has been shown above, query $\left(\operatorname{gr}(P), S_{0}\right)$ returns $Q_{0}=$ acceptable_time $\left(T_{0}\right)$. Function c_solve $\left(\operatorname{gr}\left(P_{D}\right), Q_{0}\right)$ returns true together with a collection of constraints, say, $C_{0}=10 \leq T_{0} \wedge T_{0} \leq 20$. For every $t_{0}$ satisfying $C_{0}$, atom acceptable_time $\left(t_{0}\right)$ belongs to an answer set of $\operatorname{gr}(P)$ compatible with $S_{0}$. The only regular atom undecided by $S_{0}$ is $\neg \operatorname{occur} s(a, 1)$. Suppose that $\mathcal{A C}$ solver selects this atom and calls $\mathcal{A C}$ solver $\left(P, S_{1}\right)$ where $S_{1}=S_{0} \cup\{\neg \operatorname{occurs}(a, 1)\}$. Using rule (12) of $\operatorname{gr}(P)$, function $C n\left(\operatorname{gr}\left(P_{R} \cup P_{M}\right), S_{1}\right)$ returns $S_{2}=S_{1} \cup$ $\{$ not occurs $(a, 1)\}$. Function query $\left(\operatorname{gr}(P), S_{2}\right)$ returns
$Q_{1}=$ acceptable_time $\left(T_{0}\right) \wedge$ not acceptable_time $\left(T_{1}\right)$. The possible answer returned by $c_{-}$solve $\left(\operatorname{gr}\left(P_{D}\right), Q_{1}\right)$ may be, say, $C_{1}=10 \leq T_{0} \leq 20 \wedge T_{1}<10 \wedge 20<$ $T_{1}<100 \wedge 120<T_{1}$. Finally, $\mathcal{A C}$ solver $(\operatorname{gr}(P), \emptyset)$ returns $S_{2}$. (Of course the slightly modified solver can also return $C_{1}$, or even some of its solutions). If $\mathcal{A C}$ solver were to select not $\neg \operatorname{occurs}(a, 1)$ instead of $\neg \operatorname{occurs}(a, 1)$, then the returned value would be $S_{3}=S_{0} \cup\{$ not $\neg$ occurs $(a, 1)$, not $\operatorname{occurs}(a, 1)\}$.

The algorithm is only correct for programs satisfying a number of safety conditions. In particular the usual safety conditions required for correctness of ASP and constraint solvers should be expanded by the following requirement: every middle rule of a program contains a mixed literal, and every constraint variable occurring in a middle rule of the program should occur in a mixed predicate from this rule. The following example shows why the condition is necessary. Consider a program

$$
\begin{aligned}
& \text { \#csort }(s) . \\
& \text { \#defined } d(s), e(s) . \\
& s(0 . .2) . \\
& p \leftarrow e(Y) . \\
& d(1) . \quad d(2) . \\
& e(Y) \leftarrow d(Y), Y<2 .
\end{aligned}
$$

Every answer set of this program contains $d(1), d(2)$, and $p$. Our algorithm however may also return sets not containing $p$. This happens when the algorithm picks literal not $p$, and $c_{-}$solve returns, say, $Y=0$.

\section{Representing Knowledge in $\mathcal{A C}(\mathcal{C})$}

Several examples presented in this section are meant to illustrate the use of $\mathcal{A C}(\mathcal{C})$ for knowledge representation. None of the examples can successfully run with traditional ASP solvers while all of them are easily solvable by the $\mathcal{A C}(\mathcal{C})$ solvers.

We start with a simple planning and scheduling example:

Example 2 [Planning and Scheduling]

John, who is currently at work, needs to be in his doctor's office in one hour carrying the insurance card and money to pay for the visit. The card is at home and money can be obtained from the nearby ATM. John knows the minimum time (in minutes) needed to travel between the relevant locations. Can he find a plan to make it on time? (assuming of course that there will be no delays and the actual time of travel will be the minimum time). To solve the problem we will divide it into two parts: planning and scheduling. The former can be solved by standard ASP based methods. We use variables $P$ for people, $L$ for locations, $O$ for objects (cash and the insurance card), and $S$ for steps of the planned trajectory. We will need an action go_to $(P, L)$ and fluents at_loc $(P, L)$, at_loc $(O, L)$, and has $(P, O)$ with selfexplanatory intuitive meaning. (To simplify the solution we assume that person $P$ automatically gets the 
object $O$ when both, $P$ and $O$, share the same location). The transition diagram whose states are collections of fluents describing possible physical states of the domain and arcs are labeled by actions is defined by causal laws written as logic programming rules. For instance, direct effects of the actions are described by the rule

holds $\left(\operatorname{at} \_l o c(P, L), S 1\right) \leftarrow$ $\operatorname{next}(S 1, S 0)$, occurs (go_to $(P, L), S 0)$.

Indirect effects will be captured by the corresponding relationships between fluents:

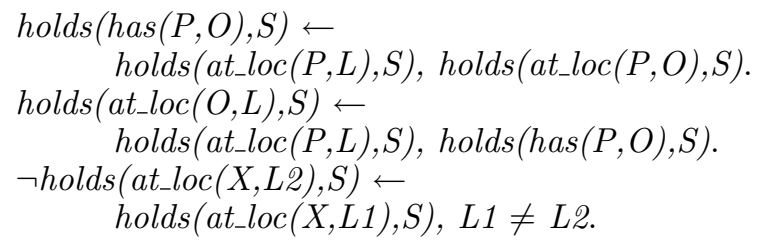

The problem of representing the unchanged fluents is solved by the inertia axioms (variable $F$ is used for fluents):

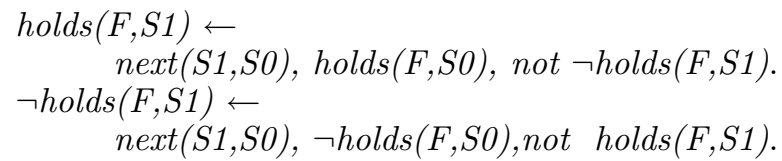

John's goal and his options will be described by the rules:

occurs $($ go_to $(j o h n, L), S)$ or $\neg$ occurs $($ go_to $(j o h n, L), S)$.

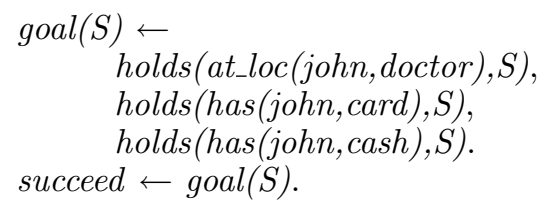

Let us denote the above program with initial conditions and sort step defined as a collection of integers from 0 to $n$ by $D_{r}^{n}$. Normally ASP planning is performed by computing answer sets of $D_{r}^{n}$ for $n=1,2, \ldots$. The desired plans can be easily extracted from the answer sets of the first consistent program $D_{r}^{k}$. A possible plan returned by this planning method can be, say, [go_to(john,home), go_to(john,atm),go_to(john,doctor)].

Now we concentrate on the scheduling part of the problem. Actual time will range from 0 to 1440 (number of minutes in 24 hours). The schedule should assign time $T$ to each step $S$ of the plan. This will be achieved by introducing a mixed relation $a t(S, T)$ and specifying the necessary constraints, e.g.,

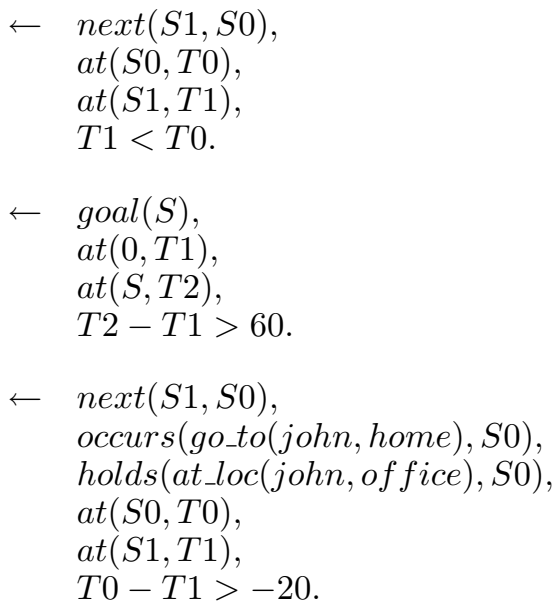

The first rule requires time to be a monotonic function of steps; the second guarantees that the trip does not take more than an hour; the third assumes that the trip from office to home takes at least twenty minutes. Other constraints are added in a similar fashion. Let us denote the resulting program by $D^{n}$.

Given this program the $\mathcal{A C}$ solver will return an answer set of $D^{n}$, containing a plan, say [occurs (go_to(john,home),0), occurs(go_to(john,atm),1), occurs(go_to(john,doctor),2)] and a schedule, say, at $(0,0)$, at $(1,20)$, at $(2,35)$, at $(3,55)$ for executing its actions. It is guaranteed that if John performs the corresponding actions as scheduled he will get to see his doctor on time. If there is no plan of length $n$ satisfying the desired conditions the program $D^{n}$ has no answer sets. The primitive constraints which occur in the middle rules of $D^{n}$ are so called difference constraint. We have an implementation of $\mathcal{A C}$ solver which works for the language $\mathcal{A C}(\mathcal{C})$ parametrized by such constraints. The current implementation, Surya $a_{d}$, has other limitations - it does not allow defined predicates and regular predicates in the heads of middle rules. Of course $D^{n}$ satisfies this condition and Surya $a_{d}$ returns the answers almost instantaneously.

In our next example we illustrate the use of $\mathcal{A C}(\mathcal{C})$ extended by consistency restoring rules of CR-Prolog.

Example 3 [Planning with Weak Constraints]

Let us now consider a variant of the story from Example 2 in which the requirement "the trip does not take more than an hour" is replaced "the trip does not take more than an hour, but John prefers to make it in 50 minutes".

The new information can be encoded by the defeasible rule which says that under normal circumstances the trip will be made in 50 minutes (or less).

$$
\begin{aligned}
\leftarrow \quad & \operatorname{goal}(S), \\
& \operatorname{at}(0, T 1), \\
& \operatorname{at}(S, T 2), \\
& T 2-T 1>50, \\
& \text { not ab(S). }
\end{aligned}
$$


The CR-rule

$a b(S) \leftarrow+\operatorname{step}(S)$.

allows, when necessary, consider exceptions to this rule. If John can get to the doctor's office in 50 minutes the program will find the corresponding plan and a proper schedule for its actions. If time constraints of the problem require time from interval $(50,60]$ the CR-rule will be used to defeat the weak constraint above and find a possible plan, say, requiring 55 minutes. The desired solutions can be easily found by Surya . $_{\text {. }}$

\section{Example 4 [Using Defined Predicates]}

Let us now consider an extension of the John's problem from Example 2 by assuming that John always travels in a cab and that the cab rate is $\$ 2.45$ per minute. John knows the amount of money in his bank account as well as the amount he needs to pay to the doctor. Now he needs not only get to the doctor on time and ready but also make sure that he has enough money for the visit. To solve the problem one may try to encode this knowledge by the following rules:

bank_account $(j o h n, 200)$.

doctor_payment(130).

taxi_rate $(2.45)$.

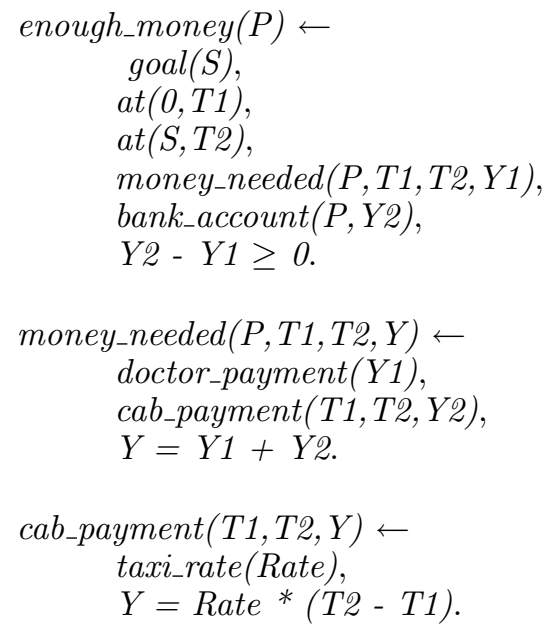

Since at the final state of the trajectory John should have enough money to pay the doctor and the taxi driver, we expand this program by a rule

$\leftarrow$ enough_money $(j o h n)$.

It is natural to declare enough_money as a regular predicate, and the rest, except the mixed predicate at and the primitive constraints, are defined predicates. It is not difficult to check that the resulting program, $E$, has an answer set and that any answer set of this program will contain enough_money (john). Notice however that $\mathcal{A C}$ solver will not be able to compute answer sets of $E$ because the definition of enough_money $(P)$ violates the safety condition of $\mathcal{A C}$ solver. The problem can be remedied by:

(a) Introducing a new defined relation $d$ specified by the rule

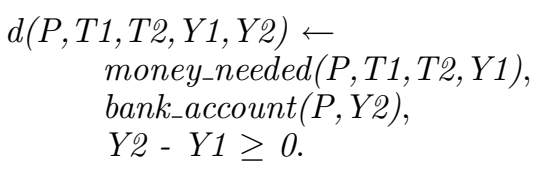

(b) Introducing a new mixed relation $m_{d}(P, Y 1, Y 2)$ whose parameters are obtained from parameters of $d$ by dropping those constraint parameters which occur in the mixed predicates of the middle rule of enough_money.

(c) Replacing the definition of enough_money by the new rule

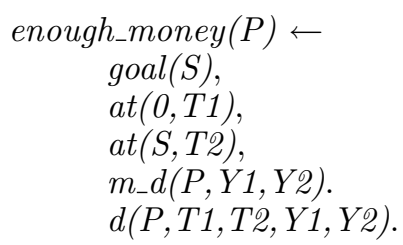

It is not difficult to show that the transformed program is equivalent to the original one modulo the new predicates. The safety condition now is satisfied and $\mathcal{A C}$ solver can be used to find the answer.

The new program has defined predicates, regular predicates in the heads of middle rules and linear constraints and thus can not be run on Surya . However, we have a second prototype implementation, $\mathcal{A C}$ engine, whose (c_solve) algorithm is implemented via constraint logic programming system CLP(R) (Jaffar et al. 1992) with constructive negation (Stuckey 1991) which is suitable for solving this problem. The only change necessary to satisfy syntactic restrictions of $\mathcal{A C}$ engine consists in replacing disjunction $p$ or $\neg p$ by two rules $p \leftarrow$ not $\neg p$ and $\neg p \leftarrow$ not $p$.

\section{Conclusion}

In this paper we introduced a knowledge representation language $\mathcal{A C}(\mathcal{C})$ extending the syntax and semantics of ASP and CR-Prolog, gave some examples of its use for knowledge representation, and presented an algorithm, $\mathcal{A C}$ solver, for computing answer sets of $\mathcal{A C}(\mathcal{C})$ programs. The algorithm does not require full grounding of a program and combines "classical" ASP solving methods with constraint logic programming techniques and CR-Prolog based abduction. The $\mathcal{A C}(\mathcal{C})$ based approach often allows to solve problems which are impossible to solve by more traditional ASP solving techniques. We believe that further investigation of the language and development of more efficient and reliable solvers for its programs can help to substantially 
expand the domain of applicability of the answer set programming paradigm. The work is based on previous results by S. Baselice, P. Bonatti, and the authors. In (Elkabani, Pontelli, \& Son 2005), an algorithm is developed to combine ASP computation with constraint solving for the purpose of reasoning with ASP aggregates. Their language however does not allow classification of predicates and hence does not avoid grounding of variables (except the variables which are local w.r.t. the aggregates). Interesting line of work investigates ways of replacing ASP programs by the corresponding constraint programs (see for instance (Dovier, Formisano, \& Pontelli 2007)). We hope that our approach will prove more attractive from the standpoint of knowledge representation and also more efficient but this is of course a matter for further research. There is also a substantial amount of work on the development of a generalization of ASP by rules which allows arbitrary "constraints atoms" (Marek \& Truszczynski 2004; Liu et al. 2007). It remains to be seen if work on the development of $\mathcal{A C}(\mathcal{C})$ solvers can profit from insights from this work.

\section{Acknowledgments}

This work was supported in part by NASA contract NASA-NEG05GP48G and ATEE/DTO contract ASU06-C-0143.

\section{References}

Apt, K., and Bezem, M. 1991. Acyclic programs. New Generation Computing 9(3,4):335-365.

Babovich, Y., and Maratea, M. 2004. Cmodels-2: SAT-based answer set solver enhanced to non-tight programs. In International Conference on Logic Programming and Nonmonotonic Reasoning, LPNMR-05.

Balduccini, M., and Gelfond, M. 2003. Logic Programs with Consistency-Restoring Rules. In Doherty, P.; McCarthy, J.; and Williams, M.-A., eds., International Symposium on Logical Formalization of Commonsense Reasoning, AAAI 2003 Spring Symposium Series, 9-18.

Balduccini, M.; Gelfond, M.; and Nogueira, M. 2006. Answer set based design of knowledge systems. Annals of Mathematics and Artificial Intelligence 47:183-219.

Balduccini, M. 2004. USA-Smart: Improving the Quality of Plans in Answer Set Planning. In $P A D L ' 04$, Lecture Notes in Artificial Intelligence (LNCS).

Balduccini, M. 2007. CR-MODELS: An inference engine for CR-Prolog. In Baral, C.; Brewka, G.; and Schlipf, J., eds., Proceedings of the 9th International Conference on Logic Programming and Non-Monotonic Reasoning (LPNMR'O'7), volume 3662 of Lecture Notes in Artificial Intelligence, 18-30. Springer.

Baral, C.; Chancellor, K.; Tran, N.; Tran, N.; Joy, A.; and Berens, M. 2004. A knowledge based approach for representing and reasoning about cell signalling networks. In Proceedings of European Conference on Computational Biology, Supplement on Bioinformatics, 15-22.

Baral, C. 2003. Knowledge Representation, Reasoning, and Declarative Problem Solving. Cambridge University Press.

Baselice, S.; Bonatti, P. A.; and Gelfond, M. 2005. Towards an integration of answer set and constraint solving. In Proceedings of ICLP-05, 52-66.

Brooks, D. R.; Erdem, E.; Minett, J. W.; and Ringe, D. 2005. Character-based cladistics and answer set programming. In Proceedings of International Symposium on Practical Aspects of Declarative Languages, $37-51$.

Chen, W.; Swift, T.; and Warren, D. S. 1995. Efficient top-down computation of queries under the wellfounded semantics. Journal of Logic Programming 24(3):161-201.

Clark, K. 1978. Negation as failure. In Gallaire, H., and Minker, J., eds., Logic and Data Bases. Plenum Press. 293-322.

Dovier, A.; Formisano, A.; and Pontelli, E. 2007. An experimental comparison of constraint logic programming and answer set programming. In Proceedings of AAAI0\%, 1622-1625.

Eiter, T.; Leone, N.; Mateis, C.; Pfeifer, G.; and Scarcello, F. 1997. A deductive system for nonmonotonic reasoning. In International Conference on Logic Programming and Nonmonotonic Reasoning, LPNMR97, LNAI 1265, 363-374. Springer Verlag, Berlin.

Elkabani, I.; Pontelli, E.; and Son, T. C. 2005. Smodelsa - a system for computing answer sets of logic programs with aggregates. In LPNMR, 427-431.

Gebser, M.; Kaufman, B.; Neumann, A.; and Schaub, T. 2007. Conflict-driven answer set enumeration. In Baral, C.; Brewka, G.; and Schlipf, J., eds., Proceedings of the 9th International Conference on Logic Programming and Nonmonotonic Reasoning (LPNMR'07), volume 3662 of lnai, 136-148. Springer.

Gelfond, M., and Lifschitz, V. 1991. Classical negation in logic programs and disjunctive databases. New Generation Computing 9(3/4):365-386.

Giunchiglia, E.; Lierler, Y.; and Maratea, M. 2006. Answer set programming based on propositional satisfiability. Journal of Automated Reasoning 36:345-377. Jaffar, J., and Maher, M. J. 1994. Constraint Logic Programming. Journal of Logic Programming 19/20:503-581.

Jaffar, J.; Michaylov, S.; Stuckey, P. J.; and Yap, R. H. C. 1992. The CLP $(\mathrm{R})$ language and system. ACM Transactions on Programming Languages and Systems 14(3):339-395.

Leone, N.; Pfeifer, G.; Faber, W.; Eiter, T.; Gottlob, G.; Perri, S.; and Scarcello, F. 2006. The DLV system for knowledge representation and reasoning. ACM Transactions on Computational Logic 7:499-562. 
Lin, F., and Zhao, Y. 2004. ASSAT: Computing answer sets of a logic program by SAT solvers. Artificial Intelligence 157(1-2):115-137.

Liu, L.; Pontelli, E.; Son, T. C.; and Truszczynski, M. 2007. Logic programs with abstract constraint atoms: The role of computations. In Proceedings of ICLP-Or, $286-301$.

Marek, V. W., and Truszczynski, M. 1993. Nonmonotonic logics; context dependent reasoning. Springer Verlag, Berlin.

Marek, V. W., and Truszczynski, M. 1999. Stable models and an alternative logic programming paradigm. The Logic Programming Paradigm: a 25-Year Perspective. Springer Verlag, Berlin. 375-398.

Marek, V. W., and Truszczynski, M. 2004. Logic programs with abstract constraint atoms. In Proceedings of $A A A I 04,86-91$.

Marriott, K., and Stuckey, P. J. 1998. Programming with Constraints: an Introduction. Cambridge, MA: MIT Press.

Mellarkod, V. S., and Gelfond, M. 2007. Enhancing asp systems for planning with temporal constraints. In LPNMR 2007, 309-314.

Niemela, I., and Simons, P. 1997. Smodels - an implementation of the stable model and well-founded semantics for normal logic programs. In Proceedings of the 4th International Conference on Logic Programming and Non-Monotonic Reasoning (LPNMR'97), volume 1265 of Lecture Notes in Artificial Intelligence (LNCS), 420-429.

Niemela, I.; Simons, P.; and Soininen, T. 2002. Extending and implementing the stable model semantics. Artificial Intelligence 138(1-2):181-234.

Niemela, I. 1999. Logic programs with stable model semantics as a constraint programming paradigm. Annals of Mathematics and Artificial Intelligence 25(34):241-27.

SICStus Prolog. $2007 . \quad$ SICStus Prolog User's Manual Version 4. http://www.sics.se/isl/sicstuswww/site/index.html.

Soininen, T., and Niemella, I. 1998. Developing a declarative rule language for applications in product configuration. In Proceedings of International Symposium on Practical Aspects of Declarative Languages, 305-319.

Stuckey, P. J. 1991. Constructive negation for constraint logic programming. In LICS, 328-339.

Van Hentenryck, P. 1989. Constraint Satisfaction in Logic Programming. Cambridge, MA: MIT Press. 